\title{
PARTITION REPRESENTATIONS OF FREE LATTICES
}

\section{ALFRED W. HALES ${ }^{1}$}

I. Introduction. If $S$ is a set, we will denote by $P(S)$ the lattice of all partitions (equivalence relations) of $S$. Whitman [6] proved that any lattice $L$ is isomorphic to a sublattice of $\rho(S)$ for some $S$. His proof shows that, if $L$ is infinite, we can take $S$ to be no larger than $L$; for finite $L$, however, his proof only shows that $S$ can be taken countable. This leaves open the following (still unsolved) problem: can every finite lattice be embedded in the lattice of partitions of some finite set?

In this paper we give a "constructive" method for obtaining certain partition representations of free lattices. These representations are obtained by a variation of Whitman's original technique in [6], and make use of both Whitman's results on free lattices in [5] and a result of Jónsson's [3]. As a corollary we show that every finite sublattice of a free lattice can be embedded in the lattice of partitions of a finite set. Furthermore, the method yields some light on both the scope and limitations of Whitman's technique.

II. Preliminaries. Let $L$ be a lattice. By a weak representation of $L$ we shall mean a mapping $\phi: L \rightarrow \odot(S)$ such that $\phi$ preserves finite intersections. A representation of $L$ is a weak representation which also preserves finite unions.

If $\phi: L \rightarrow \odot(S)$ and $\Phi: L \rightarrow \odot(T)$ are weak representations, we say that $\Phi$ is an extension of $\phi$ if $S \subseteq T$ and if, for all $a, b$ in $S$ and $A$ in $L$, we have $a \sim b$ modulo $\Phi(A)$ if and only if $a \sim b$ modulo $\phi(A)$.

The following lemma of Jónsson [3, Lemma 4.1] is basic to our results.

Lemma. Let $\phi: L \rightarrow \odot(S)$ be a weak representation of $L$, let $a$ and $b$ be elements of $S$, and suppose that $a \sim b$ modulo $\phi\left(A_{1} \cup A_{2}\right)$, where $A_{1}$ and $A_{2}$ are elements of $L$. Let $x_{1}, x_{2}, x_{3}$ be distinct elements not belonging to $S$, and define $\Phi: L \rightarrow \odot\left(S \cup\left\{x_{1}, x_{2}, x_{3}\right\}\right)$ as follows: for any $A$ in $L, \Phi(A)$ is the equivalence relation implied by the equivalences of $\phi(A)$, the equivalences $a \sim x_{1}$ and $x_{2} \sim x_{3}$ (if $A \geqq A_{1}$ ), and the equivalences $x_{1} \sim x_{2}$ and $x_{3} \sim b$ (if $A \geqq A_{2}$ ).

Then $\Phi$ is a weak representation of $L$, and is an extension of $\phi$.

Received by the editor September 3, 1967 and, in revised form, August 3, 1969.

1 This research was supported in part by NSF Grant \#GP-5497. 
III. Representations of free lattices. We assume from here on a knowledge of Whitman's paper [5] on free lattices. The following theorem is our main result:

Theorem. Let $W$ be an element of a free lattice $L$, and let $\phi: L$ $\rightarrow \odot(\{a, b\})$ be defined by $a \sim b \operatorname{modulo} \phi(A)$ if and only if $A \geqq W$. Then $\phi$ has an extension $\Phi: L \rightarrow \odot(T)$ such that $\Phi$ is a representation of $L$ and $T$ is finite.

Proof. We prove (by induction on the length of $W$ ) the following stronger result: let $\phi: L \rightarrow \odot(S)$ be a weak representation of $L$ and let $a, b$ in $S$ be such that $a \sim b$ modulo $\phi(A)$ if and only if $A \geqq W$. Then $\phi$ has an extension $\Phi: L \rightarrow \odot(T)$ such that $T-S$ is finite, and such that the following properties hold:

(i) If $u \sim v$ modulo $\Phi(A \cup B)$, where $u$, $v$ lie in $(T-S) \cup\{a, b\}$, then $u \sim v$ modulo $\Phi(A) \cup \Phi(B)$.

(ii) If $u \sim v$ modulo $\Phi(A)$, where $u \in S$ and $v \in T-S$, then either $u \sim a \sim v$ or $u \sim b \sim v$ modulo $\Phi(A)$.

If $W$ has length 1 , i.e. is a generator of $L$, then we take $T=S$, $\Phi=\phi$. Property (ii) holds vacuously and property (i) holds because, in a free lattice, $A \cup B \geqq W$ implies $A \geqq W$ or $B \geqq W$ if $W$ is a generator.

Now suppose $W \equiv \bigcap_{i=1}^{N} \bigcup_{j=1}^{n(i)} W_{i j}$, where the result has been proved for all elements shorter than $W$. (We allow the case $N=1$.) We will construct a sequence of extensions of $\phi$ culminating in the desired $\Phi$. To begin with $(i=1)$, apply the lemma of the previous section to $a, b$, and $W_{11}, \cup_{j=2}^{n(1)} W_{1 j}$. The result is an extension of $\phi$ mapping $L$ to $\rho\left(S \cup\left\{x_{1}, x_{2}, x_{3}\right\}\right)$. Now successively apply the theorem (induction hypothesis) to $a, x_{1}$, and $W_{11}$; to $x_{2}, x_{3}$, and $W_{11}$; to $x_{1}, x_{2}$, and $\bigcup_{j=2}^{n(1)} W_{1 j}$; and to $x_{3}, b$, and $\bigcup_{j=2}^{n(1)} W_{1 j}$. (This is legitimate since $W_{11}$ and $\bigcup_{j=2}^{n(1)} W_{1 j}$ are shorter than $W$.) The result is a succession of extensions of $\phi$ culminating in an extension $\Phi_{1}$.

Now repeat the above process for $i=2,3, \cdots, N$, in each case using first the lemma and then using the theorem (induction hypothesis) several times. The result is a sequence $\phi, \Phi_{1}, \cdots, \Phi_{N}$ of weak representations, each an extension of the preceding one. We claim that $\Phi_{N}=\Phi$ is the desired extension of $\phi$, i.e. that properties (i) and (ii) hold for $\Phi$.

The fact that property (ii) holds for $\Phi$ is an immediate consequence of the fact that the extension of the lemma in the previous section satisfies (ii), and each application of the induction hypothesis satisfies (ii).

To check property (i) for $\Phi$, we first note that each extension by 
means of the induction hypothesis satisfies both (i) and (ii). In light of the form of the equivalences introduced in each application of the lemma, we need only verify (i) for $u=a, v=b$ to complete the proof. Then we have $A \cup B \geqq W \equiv \bigcap_{i=1}^{N} \bigcup_{j=1}^{n(i)} W_{i j}$. In a free lattice, however, this implies either that $A$ or $B \geqq W$, in which case (i) trivially holds, or that $A \cup B \geqq \bigcup_{j=1}^{n(i)} W_{i j}$ for some $i$, say $i=1$. Thus $A \cup B \geqq W_{11}$ and $\bigcup_{j=2}^{n(i)} W_{1 j}$. But then $a \sim x_{1}, x_{2} \sim x_{3}, x_{1} \sim x_{2}$, and $x_{3} \sim b$ modulo $\Phi(A \cup B)$. The validity of (i) in each application of the induction hypothesis during the construction guarantees that each of these $\sim$ 's is valid modulo $\Phi(A) \cup \Phi(B)$, so we obtain $a \sim x_{1} \sim x_{2} \sim x_{3} \sim b$ modulo $\Phi(A)$ $\cup \Phi(B)$. This finishes the proof of the theorem.

As one corollary we obtain a result first proved by Sachs [4].

COROLlaRY 1. If $W_{1} \neq W_{2}$ in a free lattice $L$, then there is a representation $\Phi: L \rightarrow P(T)$ such that $\Phi\left(W_{1}\right) \neq \Phi\left(W_{2}\right)$ and $T$ is finite.

Proof. Assume $W_{1} W_{2}$ and apply the theorem to $W_{2}$.

Corollary. Any finite sublattice of a free lattice $L$ can be embedded in the lattice of partitions of a finite set.

Proof. Apply the theorem "independently" to each element of the sublattice. The resulting representation of $L$ will be an embedding of the sublattice.

IV. Conclusion. The difficulty in applying Whitman's technique (to obtain embeddings of finite lattices in finite partition lattices) is that his construction "never stops." The above result shows how to modify the technique so that, for some lattices, the construction does stop after a finite number of steps. It would be nice if this technique could be extended to a wider class of lattices, although the author is somewhat pessimistic about this.

Finally, the author would like to mention two related problems. First, is every finite lattice a homomorphic image of some sublattice of $P(S)$ for some finite $S$ ? Secondly, if a finite lattice $L$ is embeddable in some finite $P(S)$, is the same true for all of its homomorphic images? These two questions apparently split the original unsolved problem into two equally difficult parts.

\section{REFERENCES}

1. G. Birkhoff, Lattice theory, 3rd ed., Amer. Math. Soc. Colloq. Publ., vol. 25, Amer. Math. Soc., Providence, R. I., 1967. MR 37 \#2638.

2. M. Hall, Jr., Projective planes and related topics, California Institute of Technology, 1954. MR 17, 73. 
3. B. J6nsson, On the representation of lattices, Math. Scand. 1 (1953), 193-206. MR 15, 389.

4. D. Sachs, Identities in finite partition lattices, Proc. Amer. Math. Soc. 12 (1961), 944-945. MR 24 \#A3101.

5. P. M. Whitman, Free lattices, Ann. of Math. (2) 42 (1941), 325-330. MR 2, 244.

6. - Lattices, equivalence relations and subgroups, Bull. Amer. Math. Soc. 52 (1946), 507-522. MR 8, 62.

University of California, Los Angeles 